\title{
Coexistence of large and small polarons in manganites
}

\author{
V. Cataudella, G. De Filippis and G. Iadonisi \\ INFM, Unità di Napoli, Dipartimento di Scienze Fisiche, \\ Università di Napoli I-80125 Napoli, Italy
}

(October 27, 2018)

\begin{abstract}
The interplay of the electron-phonon interaction and of the double and super exchange magnetic effects is analyzed in the $L a_{1-x} \mathrm{Ca}_{x} \mathrm{MnO} \mathrm{O}_{3}$ perovskites with $0<x<0.5$. By using an analytical variational scheme that allows to treat the electron-phonon interaction in a fully quantum manner it is shown that in the intermediate electron-phonon coupling regime a charge density instability occurs near the metal-insulator transition, induced by either temperature or hole doping. The system segregates in antiferromagnetic or paramagnetic small polarons and ferromagnetic large polarons domains characterized by different values of the lattice distortions that are satisfactory compared with those experimentally observed.
\end{abstract}


Introduction. In recent years the perovskite oxides $\mathrm{La}_{1-x} \mathrm{Ca}_{x} \mathrm{MnO} \mathrm{O}_{3}$ have become the focus of the scientific interest owing to the discovery of the colossal magneto resistance effect. These materials have been first studied in the 1950's for their peculiar phase diagram where is present a strong correlation between magnetization and resistivity 1 In particular, in the phase of colossal magneto resistance, 2 characterized by $0.2<x<0.5$, these compounds exhibit a transition from a paramagnetic insulator to a ferromagnetic metal upon cooling that has been generally explained by the double exchange mechanism originally introduced

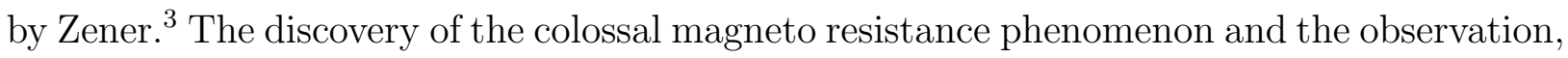
by means of the resistivity studies, 1 that the tight binding band description of the conduction electrons 3.5 is inadequate and that strong interactions between the electrons and the lattice distortions play a non negligible role have aroused a renewed interest for these compounds. The relevance of the Jahn-Teller polaron formation, first suggested by Millis, 1 has been shown by giant isotope shift of the Curie temperature, 6 by measurements of the lattice distortions by EXAFS is generally recognized. 9

Recently, a large amount of experimental data has been collected near the phase transition boundaries induced by either temperature or hole doping.10 The analysis of the experimental data showed the presence of mixed phases. For compositions intermediate near to the metal-insulator transition at low temperatures the ferromagnetic and antiferromagnetic phases coexist while the ferromagnetic and paramagnetic ones coexist in the vicinity of the paramagnetic to ferromagnetic transition temperature. On the other hands the existence of a homogeneous canted phase first proposed by de Gennes 11 in the attempt of explaining the weak ferromagnetism of the phase with $0<x<0.2$ has not been confirmed.

In these materials, the ferromagnetic phase is induced by the combined effect of the $e_{g}$ electron hopping between nearest neighbor sites and Hund's exchange with the localized $t_{2 g}$ electron spins: this phase is associated with the properties of a metallic state. On the contrary the antiferromagnetism is induced by the exchange integral between the $d$ shells of adjacent atoms and it is connected with the properties of an insulator state. 
In this paper we study, on the basis of a variational approach, the interplay of the electron-phonon interaction and of the double and super exchange magnetic effects as function of the doping and the temperature in the range $0<x<0.5$. We will show that in the intermediate electron-phonon coupling regime the combined effect of the magnetic and electron-phonon interactions pushes the system towards a charge density instability. This explains the experimentally observed tendency of the manganites to form inhomogeneous magnetic structures near the phase boundaries due to temperature or $C a$ concentrations. The metal-insulator transition in these systems is a first order transition 12 accompanied by the coexistence of two phases: a high density one made by localized small polarons forming paramegnetic or antiferromagnetic domains depending on temperature and a low density one made by itinerant large polarons forming ferromagnetic domains.

From a theoretical point of view the coexistence between hole-undoped and hole-rich phases has been discussed by using exact numerical approaches on small lattices treating the Jhan-Teller phonons classically 13 . However, little attention has been given to the quantum nature of the Jhan-Teller phonons, and, more important, to the possible formation of polarons characterizing the lattice deformations associated to the phase separation. On the other hand, after Millis et al. recognized. The main goal of the present letter is to show that the two scenario are fully compatible and that the formation of two types of polarons is responsible, together with the magnetic interactions, of the complexity of the manganites in the region $0<x<0.5$.

The model. In $L a_{1-x} C a_{x} M n O_{3}$ the electronically active orbitals are the $\mathrm{Mn} 3 d$ orbitals and the mean number of the $d$ electrons per $M n$ is $4-x$. Three electrons go into the $t_{2 g}$ core states and the remaining $1-x$ electrons occupy the outer shell $e_{g}$ orbitals. The Jahn-Teller effect splits the $e_{g}$ double degeneracy 4 and the single orbital approximation is reasonable for $x<0.5$. The corresponding Hamiltonian is given by:

$$
H=-t \sum_{i, j}\left(\frac{S_{0}^{i, j}+1 / 2}{2 S+1}\right) c_{i}^{\dagger} c_{j}+\omega_{0} \sum_{\vec{q}} a_{\vec{q}}^{\dagger} a_{\vec{q}}+\epsilon \sum_{i, j} \vec{S}_{i} \cdot \vec{S}_{j}
$$




$$
+\frac{g \omega_{0}}{\sqrt{N}} \sum_{i, \vec{q}} c_{i}^{\dagger} c_{i}\left(a_{\vec{q}} e^{i \vec{q} \cdot R_{i}}+a_{\vec{q}}^{\dagger} e^{-i \vec{q} \cdot R_{i}}\right) .
$$

Here the first term describes the double exchange mechanism, in the limit where the intra-atomic exchange integral $J$ is much greater than the transfer integral $t, S_{0}^{i, j}$ represents the total spin of the subsystem consisting of two localized spins on sites $i$ and $j$ (nearest

neighbors) and the conduction electron, $S$ is the spin of the $t_{2 g}$ core states, $\omega_{0}$ denotes the frequency of the optical local phonon mode, the dimensionless parameter $g$ indicates the strength of the electron-phonon interaction in the Holstein model, 15 t $\epsilon$ represents the antiferromagnetic super-exchange coupling between two nearest neighbor $t_{2 g}$ spins and $N$ is the number of lattice sites. The units are such that $\hbar=1$.

\section{The variational approach.}

We perform two successive canonical transformations. The first is a generalization of the Lang-Firsov unitary transformation 16 .

$$
S_{1}=\exp \left[-\frac{1}{\sqrt{N}} \sum_{j, \vec{q}} c_{j}^{\dagger} c_{j} e^{i \vec{q} \cdot \vec{R}_{j}} f_{\vec{q}}\left(a_{\vec{q}}-a_{-\vec{q}}^{\dagger}\right)\right]
$$

where the phonon distribution function $f_{\vec{q}}$ has to be determined variationally.

This choice is related to the approximation first used by Tomonaga 17 in the treatment of the coupling between mesons and nucleons and based on the assumption that all mesons are virtually emitted independently of the others. It recovers the well known Lang-Firsov 16 canonical transformation assuming narrow band small polaron, and, moreover, it provides, in the weak coupling regime, the generalization to the many-body problem of the unitary transformation utilized by Lee-Low-Pines 18 in the continuum approximation within the Frohlich model 19

The second canonical transformation is a Bogoliubov transformation. It introduces correlations between the emission of successive virtual phonons by the electron and it is responsible of the phonon frequency renormalizatione20:

$$
S_{2}=\exp \left[-\alpha \sum_{\vec{q}}\left(a_{\vec{q}}^{\dagger} a_{-\vec{q}}^{\dagger}-a_{-\vec{q}} a_{\vec{q}}\right)\right]
$$

where $\alpha$ indicates a variational parameter. 
The above introduced canonical transformations used to treat variationally the electronphonon interaction are known to give a satisfactory description of the single polaron problem in all range of couplings being able to interpolate from weak (large polaron) to strong (small polaron) interactions. For this reason we adopt the same approach here for a system of interacting polarons. However, it is worth to note that, in the present case, the utilized approach is expected to be even better than in the single polaron problem. In fact, the choice of richer trial wave function made as a linear superposition of Bloch states translationally invariant that, in the single polaron problem, gives a highly accurate description of the polaron features in the intermediate electron-phonon coupling regime, 21 does not provide any improvement in a many-polaron system since the asymptotic wave functions are orthogonal and the off-diagonal matrix elements of the Hamiltonian are zero in the thermodynamic limit.

The transformed Hamiltonian is:

$$
\begin{aligned}
\bar{H}= & -t \sum_{i, j}\left(\frac{S_{0}^{i, j}+1 / 2}{2 S+1}\right) c_{i}^{\dagger} c_{j} X_{i}^{\dagger} X_{j}+\omega_{0} \cosh 4 \alpha \sum_{\vec{q}} a_{\vec{q}}^{\dagger} a_{\vec{q}} \\
& +\frac{\omega_{0}}{\sqrt{N}} \sum_{\vec{q}, i} c_{i}^{\dagger} c_{i}\left(a_{\vec{q}}+a_{-\vec{q}}^{\dagger}\right)\left(g-f_{\vec{q}}\right) e^{i \vec{q} \cdot \vec{R}_{i}} e^{2 \alpha}+\omega_{0} \sinh 2 \alpha \cosh 2 \alpha \sum_{\vec{q}}\left(a_{\vec{q}}^{\dagger} a_{-\vec{q}}^{\dagger}+a_{-\vec{q}} a_{\vec{q}}\right) \\
& +\omega_{0} N(\sinh 2 \alpha)^{2}+\frac{\omega_{0}}{N} \sum_{i, j, \vec{q}}\left(f_{\vec{q}}^{2}-2 g f_{\vec{q}}\right) \cos \left[\vec{q} \cdot\left(\vec{R}_{i}-\vec{R}_{j}\right)\right] n_{i} n_{j}+\epsilon \sum_{i, j} \vec{S}_{i} \cdot \vec{S}_{j} .
\end{aligned}
$$

where

$$
X_{i}^{\dagger} X_{j}=\exp \left[\frac{1}{\sqrt{N}} \sum_{\vec{q}}\left(e^{i \vec{q} \cdot \vec{R}_{j}}-e^{i \vec{q} \cdot \vec{R}_{i}}\right) f_{\vec{q}} e^{-2 \alpha}\left(a_{\vec{q}}-a_{-\vec{q}}^{\dagger}\right)\right]
$$

By assuming a simple square of density of states, in the mean field approximation, the free energy per site becomes:

$$
\begin{aligned}
\frac{F}{N}= & \pm \frac{\epsilon}{2} Z S^{2} m_{S}^{2}+K_{B} T \log \left(1-e^{-\beta \omega_{0} \cosh 4 \alpha}\right)+\rho(1-\rho)\left[\frac{\omega_{0}}{N} \sum_{\vec{q}}\left(f_{\vec{q}}^{2}-2 g f_{\vec{q}}\right)-W\right] \\
& +\omega_{0}\left[(\sinh 2 \alpha)^{2}-\rho^{2} g^{2}\right]+K_{B} T\left[\rho \log \rho+(1-\rho) \log (1-\rho)+\lambda m_{S}-\log \nu_{S}\right]
\end{aligned}
$$

where the top and bottom sign, in the first term, hold, respectively, for the ferromagnetic and antiferromagnetic solutions for the localized spins. In the Eq.(6) $Z$ indicates the number of 
nearest neighbors, $\rho$ is the electron concentration, $\lambda$ is a dimensionless variational parameter that is proportional to the effective magnetic field, $\nu_{S}$ and $m_{S}$ represent, respectively, the partition function and the magnetization of the localized spins and $W$ denotes the full band width:

$$
W=Z t\left\langle\left(\frac{S_{0}+1 / 2}{2 S+1}\right)\right\rangle e^{-\frac{1}{N} \sum_{\vec{q}} f_{\vec{q}}^{2}\left[1-\cos \left(q_{x} a\right)\right]\left(2 N_{0}+1\right) e^{-4 \alpha}}
$$

where $a$ is the lattice parameter and $N_{0}$ is the average phonon number with renormalized frequency $\omega_{0} \cosh 4 \alpha$. The factor $\left\langle\left(\frac{S_{0}+1 / 2}{2 S+1}\right)\right\rangle$ gives an estimate of the reduction of the hopping due to the double exchange mechanism and it is evaluated in the ferromagnetic case by following Kubo and Ohata22 and in the antiferromagnetic case by making use of the ClebschGordan coefficients. Finally the expression of the phonon distribution function $f_{\vec{q}}$ is obtained by minimizing the free energy per site:

$$
f_{\vec{q}}=\frac{g}{1+\frac{W}{\omega_{0}} e^{-4 \alpha}\left(2 N_{0}+1\right)\left[1-\cos \left(q_{x} a\right)\right]} .
$$

The results. In the case of a single electron coupled to an optical local phonon mode we have shown 21 that a linear superposition of two Bloch wave functions describing the small and large polarons gives a very good estimate of the polaron ground state energy and provides an highly accurate description of the polaron features in the intermediate electronphonon coupling regime and for electron and phonon energy scales not well separated. In particular, in the intermediate polaron the spectral weight is equally distributed between the ground and all the excited states and the polaron wave function contains, in equal weights, the small and large polaron contributions. It is naturally to ask what happens when there are many polarons since, now, we can expect a phase separation. To this end we first perform the minimization of the free energy, subject to the condition that the total number of particles is fixed, with respect to the two variational parameters $\alpha$ and $\lambda$ by making use of the self-consistent equation for the bandwidth $W$. Then, in order to investigate the existence of coexisting phases, we study the function $G(\rho, \mu)=F(\rho)-\mu \rho$, where $F(\rho)$ is the free energy calculated from the above discussed minimization procedure and $\mu$ is 
a Lagrangian multiplier to be adjusted to get the desired total number of particles. The function $G(\rho, \mu)$, at fixed value of the temperature, is reported in Fig.1 for different values of the chemical potential $\mu$ in the intermediate electron-phonon coupling regime $\lambda_{p} \simeq 1$ ( $\lambda_{p}$ represents the ratio between the small polaron binding energy and the energy gain of an itinerant electron on a rigid lattice). From Fig.1 it is evident that there are values of the chemical potential $\mu$ such that the function $G$ is stationary for three distinct electron densities. In particular, there is a value of $\mu$ such that $G$ is the same at two different values of the electron density: $\rho_{1}$ and $\rho_{2}$. They characterize the coexistence region. We stress that $\rho_{1}$ and $\rho_{2}$ depend on the temperature and correspond to homogeneous phases of the system constituted by small and large polarons. Below a critical temperature the system segregates (see Fig.2) in antiferromagnetic or paramagnetic and ferromagnetic domains (with density $\rho_{1}$ and $\rho_{2}$ ) characterized by different values of the lattice distortions and by distinct behaviors of the resistivity with the temperature. The domains of large polarons are characterized by a weak electron mass renormalization and by coherent motion. On the other hand within the domains of small polarons the well-known band collapse takes place, the coherent motion is suppressed rapidly with increasing the temperature and the resistivity has a thermal activated behavior characteristic of the semiconducting phase.

In the region of coexistence the fractions of volume, $V_{1} / V$ and $V_{2} / V$, filled with density $\rho_{1}$ and $\rho_{2}$, are determined by the two following conditions: $V_{1} / V+V_{2} / V=1$ and $V_{1} / V \rho_{1}+$ $V_{2} / V \rho_{2}=\rho$, where $\rho=n / V$ and $n$ represents the total number of charge carriers. It is crucial, in order to confirm our results, to evaluate the lattice displacement associated to the two kinds of coexisting domains. An estimate of the average deviation of the molecule on the site $i$ from the equilibrium position when one electron is on site $i$ is given by the function:

$$
D=\frac{1}{N \rho} \sum_{i}\left\langle c_{i}^{\dagger} c_{i}\left(\frac{a_{i}+a_{i}^{\dagger}}{\sqrt{2 M \omega_{0}}}\right)\right\rangle
$$

where $M$ denotes the ionic mass.

Applying the two canonical transformations $S_{1}$ and $S_{2}$ one finds: 


$$
D=\frac{2}{\sqrt{2 M \omega_{0}}}\left[(1-\rho) \frac{1}{N} \sum_{\vec{q}} f_{\vec{q}}+g \rho\right] .
$$

In particular, for narrow band small polarons $f_{\vec{q}}$ is of order of $g$ and $D$ assumes the value $2 g / \sqrt{2 M \omega_{0}}$.

By using the following values for the model parameters: $t=2 \omega_{0}, g=3.5, \epsilon=0.01 t$, $\omega_{0}=50 \mathrm{meV}$, for $M$ the mass of the oxygen atom and by taking into account that the JahnTeller distortion of the $\mathrm{Mn}^{3+}$ ion consists in an axial elongation of the two $M n O$ bonds of the $\mathrm{MnO}_{6}$ octahedra, at $\mathrm{T}=240 \mathrm{~K}$ we find: $\mathrm{D} / 2 \simeq 0.18 \stackrel{\AA}{A}$ for the paramagnetic domains

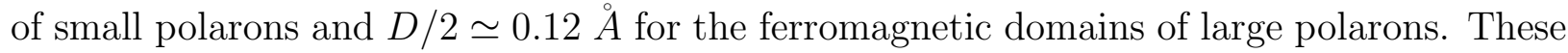
results are in agreement with the experimental datat on $\mathrm{La}_{0.75} \mathrm{Ca}_{0.25} \mathrm{MnO}_{3}$ compound, that show evidence, above $170 \mathrm{~K}$, of small polarons characterized by $\mathrm{MnO}$ bond of order of $\sim 0.22 \AA$ and large polarons with $M n O$ bond of order of $\sim 0.1 \AA$.

In summary, we have studied the combined effect of the magnetic and electron-phonon interactions in $\mathrm{La}_{1-x} \mathrm{Ca}_{x} \mathrm{MnO}_{3}$ perovskites by means of two canonical transformations and the mean field theory. It has been shown that in the intermediate electron-phonon coupling regime these systems form inhomogeneous magnetic structures near the metal-insulator transition, segregating into antiferromagnetic or paramagnetic and ferromagnetic domains of small and large polarons characterized by different values of the lattice distortions as experimentally observed.

\section{FIGURE CAPTIONS}

F1 The function $G(\rho, \mu)=F(\rho)-\mu \rho$ is plotted as a function of the doping for different values of the chemical potential at fixed temperature: $K_{B} T=0.4 \omega_{0}$ (for $\omega_{0}=50 \mathrm{meV}$ $K_{B} T=0.5 \omega_{0}$ corresponds to a temperature $\mathrm{T}$ of order of $\left.300 K\right)$.

F2 The phase diagram corresponding to: $t=2 \omega_{0}, g=3.5, \epsilon=0.01 t$. The area between the dotted lines indicates the region where the localized and delocalized phases coexist. 
${ }^{1}$ G.H. Jonker and J.H. Santen, Physica 16, 337 (1950); J.H. Santen and G.H. Jonker, Physica 16, 599 (1950).

${ }^{2}$ P. Schiffer, A.P. Ramirez, W. Bao and S.W. Cheong, Phys. Rev. Lett. 75, 3336 (1995).

${ }^{3}$ C. Zener, Phys. Rev. 81, 440 (1951); C. Zener, Phys. Rev. 82, 403 (1951).

${ }^{4}$ A.J. Millis, P.B. Littlewood and B.I. Shraiman, Phys. Rev. lett. 74, 5144 (1995); A.J. Millis, B.I. Shraiman and R. Mueller, Phys. Rev. Lett. 77, 175 (1996).

${ }^{5}$ P.W. Anderson and H. Hasegawa, Phys. Rev. 100, 675 (1955); P.G. de Gennes, Phys. Rev. 118, $141(1960)$.

${ }^{6}$ Guo-Meng-Zhao, K. Coder, H. Keller and K.A. Muller, Nature (London) 381, 676 (1996).

${ }^{7}$ A. Lanzara, N.L. Saini, M. Brunelli, F. Natali, A. Bianconi, P.G. Radaelli and S.W. Cheong, Phys. Rev. Lett. 81, 878 (1998).

${ }^{8}$ K.H. Kim, J.Y. Gu, H.S. Choi, G.W. Park and T.W. Noh, Phys. Rev. Lett. 77, 1877 (1996).

${ }^{9}$ A.J. Millis, Nature (London) 392, 147 (1998).

${ }^{10}$ G. Allodi, R. De Renzi, G. Guidi, F. Licci, and M.W. Pieper, Phys. Rev. B 56, 6036 (1997); G. Allodi, R. De Renzi, and G. Guidi, Phys. Rev. B 57, 1024 (1998); J. Dho, I. Kim, S. Lee, K.H. Kim, H.J. Lee, J.H. Jung, and T.W. Noh, Phys. Rev. B 59, 492 (1999); S.J.L. Billinge, Th. Proffen, V. Petkov, J.L. Sarrao, and S. Kycia, Cond-Mat 9907329 (1999).

${ }^{11}$ P.G. de Gennes, Phys. Rev. 118, 141 (1960).

12 J. B. Goodenough and J. S. Zhou, Nature 386, 229 (1997).

13 A. Moreo, S. Yunoki and E. Dagotto, Science 283, 2034 (1999)

${ }^{14}$ J. Zhang, A.R. Bishop, and H. Roder, Phys. Rev. B 53, 8840 (1996).

15 T. Holstein, Ann. Phys. 8, 325 (1959); T. Holstein, Ann. Phys. 8, 343 (1959). 
${ }^{16}$ I. J. Lang and Yu. A. Firsov, Soviet Physics JETP 16, 1301 (1963); Yu. A. Firsov, Polarons (Moskow, Nauka, 1975).

17 S. Tomonaga, Prog. Theor. Phys. 2, 6 (1947).

18 T.D. Lee, F. Low, and D. Pines, Phys. Rev. 90, 297 (1953).

${ }^{19}$ H. Fröhlich et al., Philos. Mag. 41, 221 (1950); H. Fröhlich, in Polarons and Excitons, edited by C.G. Kuper and G.A. Whitfield (Oliver and Boyd, Edinburg, 1963), p. 1.

${ }^{20}$ H. Zheng, Phys. Rev. B 36, 8736 (1987); H. Zheng, Solid State Communication 65, 731 (1988).

${ }^{21}$ V. Cataudella, G. De Filippis, and G. Iadonisi, Phys. Rev. B 60, 15163 (1999); V. Cataudella, G. De Filippis and G. Iadonisi, Phys. Rev. B 62, 1496 (2000).

${ }^{22}$ K. Kubo and N. Ohata, J. Phys. Soc. Jpn 33, 21 (1972). 


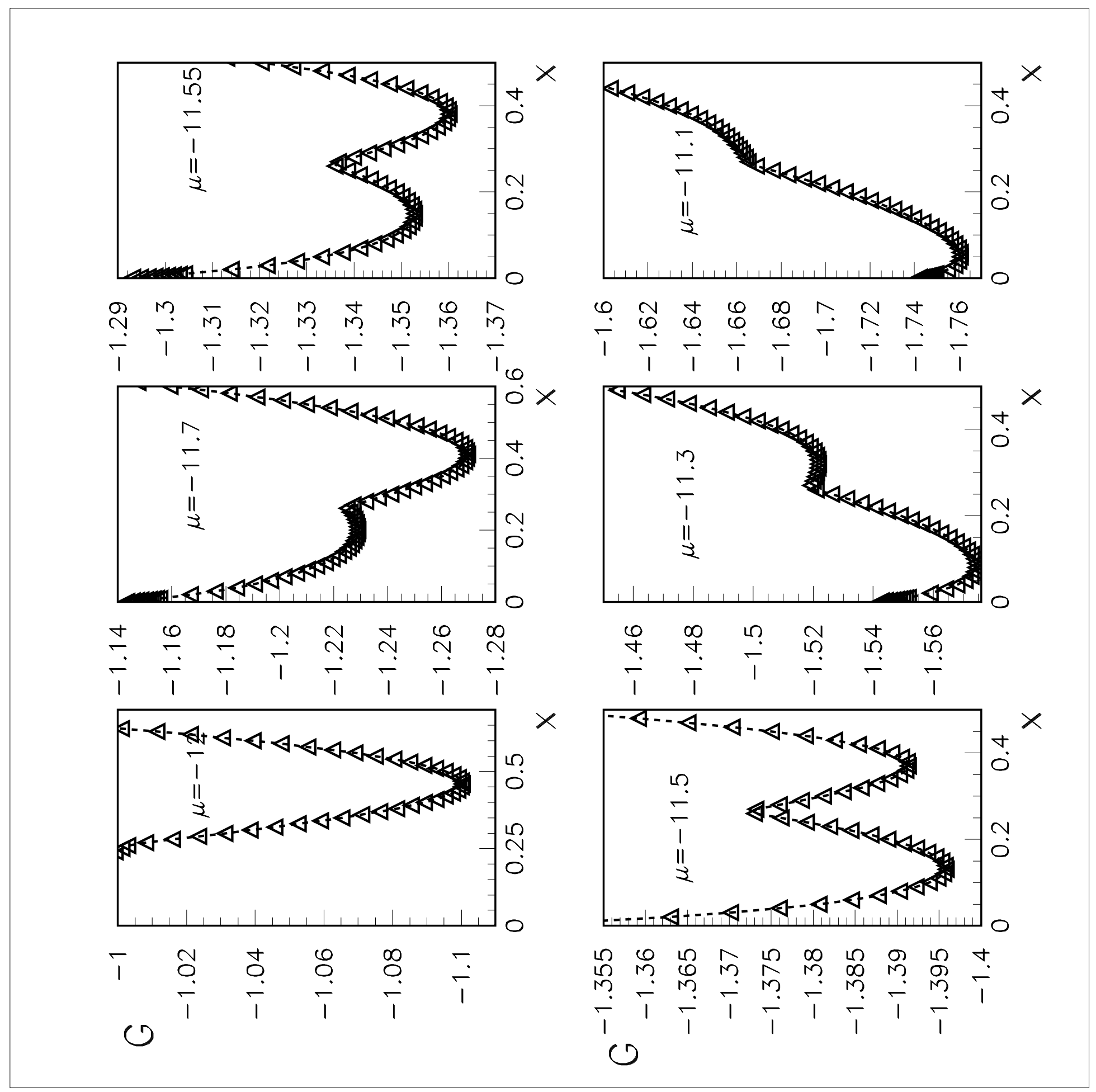




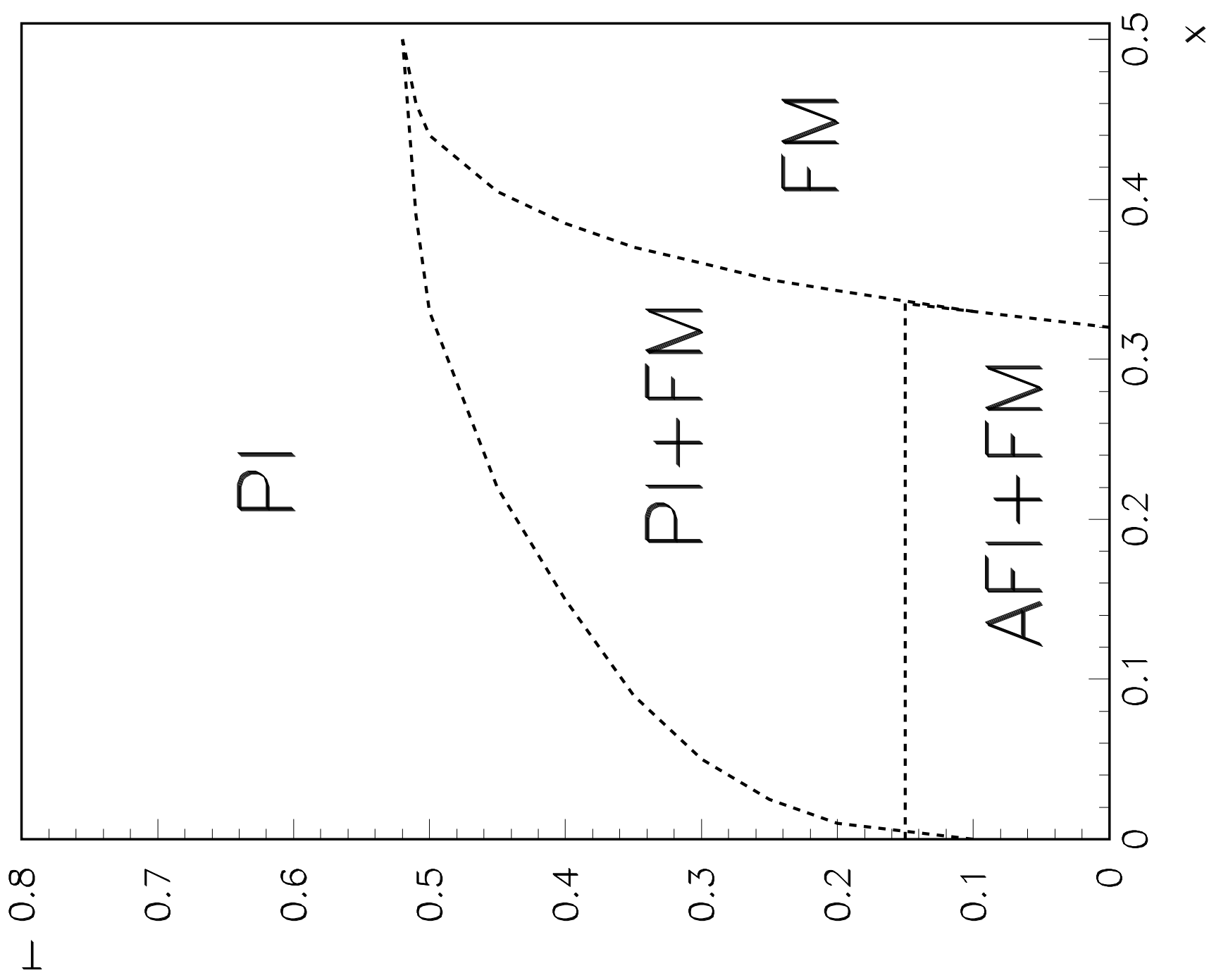

